\title{
Downregulation of gangliotetraosylceramide and $\beta 1,3$-galactosyltransferase-4 gene expression by Smads during transforming growth factor $\beta$-induced epithelial-mesenchymal transition
}

\author{
JIA GUO ${ }^{1}$, BO SONG ${ }^{1}$, XIANG LI $^{2}$, CHENHUI HE ${ }^{1}$, GANGLONG YANG ${ }^{1}$, XIAOMIN YANG $^{3,4}$ and FENG GUAN \\ ${ }^{1}$ Key Laboratory of Carbohydrate Chemistry and Biotechnology, Ministry of Education, School of Biotechnology; \\ ${ }^{2}$ Wuxi Medical School, Jiangnan University, Wuxi, Jiangsu 214122; ${ }^{3}$ Department of Oncological Surgery, \\ The First Affiliated Hospital, School of Medicine, Xi'an Jiaotong University; ${ }^{4}$ Center of Breast Oncology, \\ Shaanxi Province Tumor Hospital, Xi'an, Shaanxi 710061, P.R. China
}

Received December 17, 2013; Accepted July 28, 2014

DOI: $10.3892 / \mathrm{mmr} .2014 .2912$

\begin{abstract}
The process of epithelial-mesenchymal transition (EMT) is essential for the proliferation and metastasis of tumor cells. Our previous study demonstrated that the expression of gangliotetraosylceramide $(\mathrm{Gg} 4)$ and the transcription of UDP-Gal: $\beta 1,3$-galactosyltransferase-4 ( $\beta 3 \mathrm{GalT} 4)$, a gene which controls the expression of $\mathrm{Gg} 4$, are reduced during transforming growth factor (TGF)- $\beta$-induced EMT in normal murine mammary gland (NMuMG) cells. The present study revealed that the transcription level of $\beta 3$ GalT4 in patients with breast cancer was decreased compared with healthy control subjects. However, the molecular basis underlying these effects remains to be elucidated. Analysis of the $\beta 3$ GalT4 promoter sequence revealed a putative Smad-binding element (SBE) of $\mathrm{Smad} 4$, which is a transcriptional factor in TGF $\beta$ responses and forms a complex with Smad3. To clarify the association between the Smad3/4 complex and the $\beta 3$ GalT4 gene during EMT in NMuMG cells, an electrophoretic mobility shift assay and a chromatin immunoprecipitation assay were performed. The Smad3/4 complex was found to bind directly to the SBE of the $\beta 3$ GalT4 promoter. Overexpression of Smad3 and Smad4 through stable transfection had no notable effect on cell phenotype, but did alter the expression of various EMT protein markers. Treatment with TGF $\beta$ reduced the expression of Gg4 and the mRNA levels of the $\beta 3$ GalT4 gene in Smad3- and Smad4-overexpressing cells compared with vector-transfected
\end{abstract}

Correspondence to: Professor Feng Guan, Key Laboratory of Carbohydrate Chemistry and Biotechnology, Ministry of Education, School of Biotechnology, Jiangnan University, 1800 Lihu Avenue, Wuxi, Jiangsu 214122, P.R. China

E-mail: fengguan@jiangnan.edu.cn

Key words: gangliotetraosylceramide, $\beta 1,3$-galactosyltransferase-4, epithelial-mesenchymal transition, Smads, transcription factor cells. Expression of the epithelial markers E-cadherin and $\beta$-catenin decreased in parallel with the reduction in $\mathrm{Gg} 4$. These findings suggested that the activated Smad3/4 complex downregulated the expression of $\mathrm{Gg} 4$ and the $\beta 3$ GalT4 gene through translocation into the nucleus and binding to the $\beta 3$ GalT4 promoter.

\section{Introduction}

Glycosphingolipids (GSLs), consisting of a hydrophobic ceramide backbone and a hydrophilic carbohydrate residue, are an important type of glycolipid found in all animal cell surface membranes. GSLs are important in a variety of normal physiological processes and pathological conditions, including embryogenesis, immune responses, signal transduction as well as tumor initiation and progression (1-6). However, the mechanisms underlying the regulation of GSL biosynthesis remain to be elucidated.

Previous studies have demonstrated that epithelial-mesenchymal transition (EMT) is an important process in disease development, particularly in tumor metastasis. During EMT, cells undergo a morphological change from epithelial cells, arranged in a cobblestone-like monolayer, to dispersed mesenchymal cells, which are spindle-shaped. They also exhibit a reduction in the expression of epithelial cell marker molecules, including E-cadherin (E-cad) and an increase in the expression of mesenchymal cell marker molecules, including fibronectin, $\mathrm{N}$-cadherin (N-cad) and vimentin. Furthermore, they exhibit enhanced motility, enabling them to invade neighboring tissues through the extracellular matrix $(7,8)$. Transforming growth factor- $\beta$ (TGF $\beta$ ) is commonly used to induce EMT in cancer cell models. Our previous study demonstrated a decrease in the expression of gangliotetraosylceramide $(\mathrm{Gg} 4)$ and in the transcription of the $\beta 1,3$-galactosyltransferase- 4 ( $\beta 3$ GalT4) gene during a TGF $\beta$-induced EMT process in normal murine mammary gland (NMuMG) cells (9). The expression of Gg4 and the key epithelial markers E-cad and $\beta$-catenin reduced with a similar time course during EMT. Immunoprecipitation 
assays confirmed the interaction between Gg4, E-cad and $\beta$-catenin and suggested that the EMT process is modulated by $\mathrm{Gg} 4$ through its interaction with E-cad and $\beta$-catenin at the NMuMG cell surface $(9,10)$. However, the molecular mechanism underlying the reduced gene transcription of $\beta 3$ GalT4 during TGF $\beta$-induced EMT remains to be elucidated.

TGF $\beta$ signal transmission involves numerous biochemical pathways, including the TGF $\beta /$ Smads signaling pathway (11). In this pathway, TGF $\beta$ activates receptor-regulated SMAD (R-Smads) proteins, Smad2 and Smad3, through receptor-induced phosphorylation, thereby reducing the affinity of R-Smads for cytoplasmic anchors and increasing their accumulation in the nucleus (11-13). R-Smads and Smad4 located in the nucleus recruit co-activators/repressors and bind to the promoters of target genes to regulate their transcription (14). The Smad3/4 complex can bind directly to a DNA sequence (5'-GTCT-3') termed the Smad-binding element (SBE) $(15,16)$. Analysis of the $\beta 3$ GalT4 promoter has revealed a number of potential binding sites for transcription factors, including a Smad4-binding site (5'-GTCTAGAC-3') (17). However, to the best of our knowledge, there is no experimental evidence that the reduced gene expression of $\beta 3$ GalT4 by TGF $\beta$ is regulated via Smads in general or in particular via the Smad3/4 complex.

In the present study, the transcription levels of the $\beta 3$ GalT4 gene was detected in patients with breast cancer, and the association between Smad3/4 and Gg4 during the EMT process was further studied.

\section{Materials and methods}

Cell lines, culture and samples. NMuMG epithelial cells were obtained from the American Type Culture Collection (Manassas, VA, USA) and cultured in Dulbecco's modified Eagle's medium (DMEM; HyClone, Logan, UT, USA) containing $10 \%$ fetal bovine serum (HyClone), $10 \mu \mathrm{g} / \mathrm{ml}$ insulin (Sigma-Aldrich, St. Louis, MO, USA) and $1 \mathrm{X}$ penicillin/streptomycin (Gibco-BRL, Carlsbad, CA, USA) at $37^{\circ} \mathrm{C}$ in $5 \% \mathrm{CO}_{2}$.

All samples from the patients with breast cancer and the healthy controls were collected at the First Affiliated Hospital of Xi'an, Jiaotong University (Xi'an, China). The patient characteristics are shown in Table I. The present study followed the tenets of the Declaration of Helsinki for the Use of Human Subjects. The present study was approved by the Ethics Committee of Jiangnan University (Wuxi, Jiangsu, China) and written informed consent was obtained from all patients and healthy donors.

\section{Antibodies and reagents}

Antibodies. Mouse anti-Gg4 immunoglobulin (Ig)M monoclonal antibody (mAb) TKH7 (Kjeldsen and Hakomor, unpublished data) was donated by Dr S. Hakomori (Biomembrane Institute, Seattle, WA, USA). The primary antibodies used were mouse anti-E-cad IgG2a monoclonal antibody and mouse-anti- $\beta$ catenin IgG1 monoclonal antibody (BD Biosciences, San Jose, CA, USA), mouse anti-N-cad IgG1 and rabbit anti-RNA polymerase II ( $\mathrm{Pol}$ II) IgG polyclonal antibody (Santa Cruz Biotechnology, Inc., Santa Cruz, CA, USA), mouse anti-vimentin IgG1 monoclonal antibody and anti- $\beta$ tubulin IgG1 monoclonal antibody (Sigma-Aldrich), rabbit anti-Smad3 $\mathrm{mAb}$ IgG monoclonal antibody and anti-Smad4 polyclonal antibody (Cell Signaling Technology, Boston, MA, USA). The secondary antibodies used were horseradish peroxidase (HRP)-labeled goat anti-mouse IgG and HRP-labeled goat anti-rabbit IgG (Beyotime Institute of Biotechnology, Haimen, China) and HRP-labeled goat anti-mouse IgM (Southern Biotech, Birmingham, Alabama, USA).

Reagents. TGF $\beta$ was obtained from BD Biosciences and Asialo-ganglio-N-tetraosylceramide (asialo-GM1; Gg4), GM1, GM2, GM3 and GM4 were obtained from Matreya Inc. (Pleasant Gap, PA, USA). Other reagents used were obtained from Sigma-Aldrich, unless described otherwise.

Expression of recombinant hexahistidine $\left(\mathrm{His}_{6}\right)$-tagged Smad3 and Smad4 proteins in Escherichia coli (E. coli). The following primers were used to amplify the gene for mouse Smad3: Smad3p28, forward 5'-GGAATTTCATATTCGTCCATC CTGCCC-3', NdeI and Smad3cDNA, reverse 5'-CCGCTCGAG ACCCGCTCCCTTTACTCCTA-3', XhoI. The following primers were used were used to amplify the gene for mouse Smad4: Smad4p28, forward 5'-GGAATTTCATATGGACAA TATGTCTATAACAAATAC-3', NdeI and Smad4cDNA, reverse 5'-GGAATTCCTGAGATCTCAGTCTAAAGGCT-3', EcoRI. The polymerase chain reaction (PCR) fragments were inserted into the expression vector pET-28a $(+)$ to generate pET28-Smad3 and pET28-Smad4 and introduced into $E$. coli BL21 (DE3) for protein expression. The recombinant $\mathrm{His}_{6}$-tagged Smad3 and Smad4 proteins produced by isopropyl $\beta$-D-1-thiogalactopyranoside induction were purified on a $\mathrm{Ni}^{2+}$-NTA spin column (Roche Diagnostics, Basel, Switzerland).

Electrophoretic mobility shift assay (EMSA). EMSAs were performed using a 2nd Generation DIG Gel Shift kit (Roche Diagnostics) according to the manufacturer's instructions. The probes were amplified using PCR and labeled with digoxigenin (DIG) at the 3'-terminal end. The probes, proteins and poly(deoxyinosinic-deoxycytidylic) in binding buffer $[100 \mathrm{mM}$ Hepes, $\mathrm{pH}$ 7.6; 5 mM EDTA; $50 \mathrm{mM}\left(\mathrm{NH}_{4}\right)_{2} \mathrm{SO}_{4} ; 5$ mM DTT; Tween 20, 1\% (w/v); 150 mM KCl; Roche Diagnostics, Basel, Switzerland] were mixed and incubated at $25^{\circ} \mathrm{C}$ for $30 \mathrm{~min}$ prior to adding $5 \mu \mathrm{l}$ loading buffer. The protein-DNA complex and free DNA were separated on native $5 \%$ polyacrylamide gels and transferred onto nylon membranes. The membranes were treated according to the manufacturer's instructions and the bands on the membranes were detected using a Chemi Doc XRS chemiluminescent imaging system (Bio-Rad, Hercules, CA, USA).

Chromatin immunoprecipitation (ChIP) assay. ChIP assays were performed, as described previously (18). In brief, NMuMG cells were grown in regular medium (DMEM medium containing $10 \%$ fetal bovine serum) for $24 \mathrm{~h}$, following which the medium was replaced by regular medium containing $2 \mathrm{ng} / \mathrm{ml} \mathrm{TGF} \beta$ and the cells were incubated for $48 \mathrm{~h}$ at $37^{\circ} \mathrm{C}$ and then fixed in DMEM containing $1 \%$ formaldehyde. ChIP was performed using the anti-Smad4 antibody. Anti-Pol II antibody immunoprecipitated with a Pol II-actin promoter complex was used as a positive control. DNA was extracted and pellets 
Table I. Patient/disease characteristics and level of $\beta 3$ GalT4 mRNA in patients with breast cancer.

\begin{tabular}{|c|c|c|c|c|}
\hline Sample no. & Age (years) & Tumor size (cm) & $\mathrm{TNM}^{\mathrm{a}}$ classification & $\begin{array}{l}\text { } 33 \text { GalT4 expression } \\
\text { (relative fold change) }\end{array}$ \\
\hline \multicolumn{5}{|c|}{ Healthy donors } \\
\hline Normal 1 & 49 & - & - & 1 \\
\hline Normal 2 & 54 & - & - & $1.00 \pm 0.237$ \\
\hline Normal 3 & 35 & - & - & $1.26 \pm 0.174$ \\
\hline \multicolumn{5}{|c|}{ Cancer samples } \\
\hline 1 & 54 & multifocal & T2N3M0 IIIc & $0.04 \pm 0.005^{\mathrm{c}}$ \\
\hline 2 & 39 & 1.6 & T1N0M0 I & $0.26 \pm 0.055^{\mathrm{b}}$ \\
\hline 3 & 35 & 2.3 & T2N1M0 IIb & $0.09 \pm 0.005^{\mathrm{c}}$ \\
\hline 4 & 60 & 6.0 & T4N1M0 IIIb & $0.18 \pm 0.006^{c}$ \\
\hline 5 & 57 & 2.5 & T2N0M0 IIa & $0.04 \pm 0.007^{\mathrm{c}}$ \\
\hline 6 & 33 & 2.0 & T1N1M0 IIa & $0.55 \pm 0.053^{b}$ \\
\hline 7 & 42 & 2.5 & T2N1M0 IIb & $0.02 \pm 0.003^{c}$ \\
\hline 8 & 50 & 2.0 & T1N3M0 IIIc & $0.17 \pm 0.045^{\mathrm{c}}$ \\
\hline \multicolumn{5}{|c|}{ 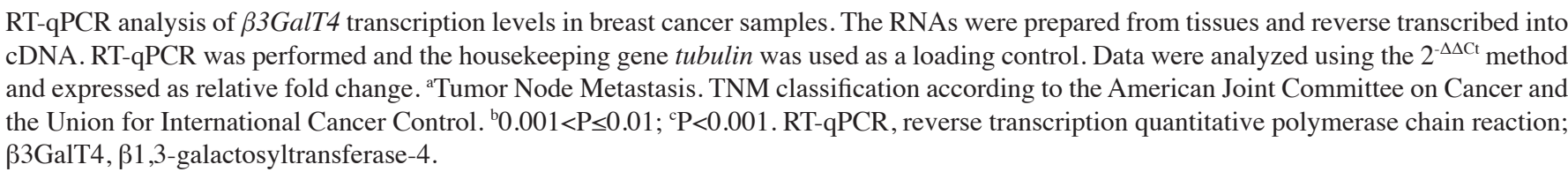 } \\
\hline
\end{tabular}

were purified using a Quick DNA purification kit (Beijing CoWin Biotech. Co., Beijing, China). The DNA template $(2 \mu \mathrm{l})$ was used for PCR with the following primers: $\beta 3 \mathrm{GalT} 4 \mathrm{pL}$, forward 5'-GGTGTGTTAGGGGACTGGT-3' and reverse 5'-TGGACTGTGCAGCCTGAT-3' for the $\beta 3$ GalT4 promoter; pNon, forward 5'-CTGAGGGTCTTGAGGGTGAG-3' and reverse 5'-CTCTTCCTCCTGGGAAAACC-3' for the nonspecific DNA fragment and pactinp forward 5'-TCAATCTCGCTTTCTCTCTCG-3' and reverse 5'-CAA CGAAGGAGCTGCAAAG-3' for the actin promoter.

Cell transfection. The protein coding regions of the genes were amplified by PCR using the following primers: Smad3cDNA, forward '5-CCCAAGCTTGCCACCATGTCGTCCATCCT GCCC-3', HindIII, and Smad3cDNA, reverse for the smad3 gene. Smad4cDNA, forward '5-GGGGTACCCCCTTGAACA AATGGACAATATGT-3', KpnI, and Smad4cDNA, reverse for the smad4 gene. The products were digested and ligated into vector pcDNA3.1 (Invitrogen Life Technologies, Carlsbad, CA, USA). The constructed plasmids were transfected into NMuMG cells using Lipofectamine 2000 (Invitrogen Life Technologies). Stable transfectants were selected by screening with the antibiotic G418 and confirmed by western blot analysis.

Western blot analysis. Cells were harvested and lysed in radioimmunoprecipitation assay buffer. Protein lysates were analyzed by SDS-PAGE and western blot analysis, as described previously (10).

Semiquantitative and quantitative reverse transcription (RT)-PCR analysis. RNA was extracted using an RNApure Tissue kit (Beijing CoWin Biotech, Co.). RNA samples were treated with DNase I and assessed by PCR to rule out chromosomal DNA contamination. Each RNA sample was reverse transcribed using ReverTra Ace- $\alpha-{ }^{\circledR}$ (Toyobo, Shanghai, China). Semiquantitative and quantitative RT-PCR analysis were performed to determine the transcription levels of various genes using the following primers: $\beta 3 \mathrm{GalT4}$ real, forward 5'-CTCTTCCTCCTGGGAAAACC-3' and reverse 5'-CTGAGGGTCTTGAGGGTGAG-3' for the $\beta 3$ GalT4 gene and Tubulinreal, forward 5'-ATCTACCTGTCGGAGCATGG-3' and reverse 5'-GCCTCCCGATCTATGATGTC-3' for the Tubulin gene. The following thermocycler conditions were used for RT-qPCR: $95^{\circ} \mathrm{C}$ for $10 \mathrm{~min}, 40$ cycles of $95^{\circ} \mathrm{C}$ for $10 \mathrm{sec}$ and $60^{\circ} \mathrm{C}$ for $1 \mathrm{~min}$ in a $15 \mu \mathrm{l}$ reaction system using an UltraSYBR mixture (Beijing CoWin Biotech, Co.). The DNA products were analyzed using CFX manager software (version 3.0.1224.1015; Bio-Rad).

GSL extraction, analysis and immunostaining. GSL extraction, high performance thin layer chromatography (HPTLC) analysis and immunostaining were performed, as described previously (19). Cells were harvested following washing with PBS, extracted with $2 \mathrm{ml}$ isopropanol/hexane/water (55:25:20) by sonication for $30 \mathrm{~min}$ and centrifuged at $1082 \mathrm{x}$ for $5 \mathrm{~min}$. The extracts were dried in a nitrogen stream. Redissolved GSLs were incubated in $0.1 \mathrm{M} \mathrm{NaOH}$ in methanol at $40^{\circ} \mathrm{C}$ for $2 \mathrm{~h}$ to hydrolyze the phospholipids and then neutralized with $1 \mathrm{M}$ $\mathrm{HCl}$. The hydrolyzed phospholipids were removed by hexane and the remaining solution was evaporated and dissolved in $1 \mathrm{ml}$ distilled water. The solution was applied to a Sep-Pak C18 cartridge (Varian Medical Systems, Palo Alto, CA, USA) and washed with water. The total GSLs were eluted with $2 \mathrm{ml}$ chloroform/methanol (2:1) and stained using $0.5 \%$ orcinol in 
$2 \mathrm{M}$ sulfuric acid. $\mathrm{Gg} 4$ bands were then immunostained using mAb TKH7 on HPTLC plates (EMD Biosciences, Inc., San Diego, CA, USA).

Statistical analysis. Data were analyzed using a two-tailed two-sample t-test assuming equal variance using GraphPad Prism 5 software (GraphPad Software Inc., La Jolla, CA, USA). $\mathrm{P} \leq 0.05$ was considered to indicate a statistically significant difference.

\section{Results}

Expression of $\beta 3$ GalT4 at the mRNA level in breast cancer and healthy control subjects. Our previous study demonstrated that the transcription levels of $\beta 3$ GalT4 were decreased during TGF $\beta$-induced EMT in NMuMG cells (9). To determine the expression of $\beta 3$ GalT4 in breast cancer, the expression of $\beta 3$ GalT4 at the mRNA level in different clinical samples was determined using RT-qPCR. As shown in Table I, a significant reduction in the expression of $\beta 3$ GalT4 was detected in the patients with breast cancer compared with the healthy control subjects. This suggested that decreased expression of the $\beta 3$ GalT4 gene is often present during breast cancer progression.

Interaction between Smad3/4 and the $\beta 3$ GalT4 promoter. The Smad3 and Smad4 proteins have a 'Mad-homology 1' domain, a DNA-binding module stabilized by a bound zinc atom, at the N-terminus (11). Smad3 and Smad4 can bind to DNA directly via the SBE (16). A previous study of the $\beta 3$ GalT4 promoter (17) revealed a Smad4 binding site between positions -788 and -795 (5'-GTCTAGAC-3') relative to the $\beta 3$ GalT4 transcriptional start point (Fig. 1A). In the present study, EMSAs and ChIP assays were performed to identify the interactions between Smads and the $\beta 3$ GalT4 promoter.

EMSAs were performed using full-length recombinant His $_{6}$-Smad 3 and - Smad4 proteins expressed in E. coli. The probe used was a 237 bp DNA fragment of the $\beta 3$ GalT4 promoter, amplified using the forward and reverse $\beta 3 \mathrm{GalT} 4 \mathrm{pL}$ primers and labeled with DIG. The probe was clearly retarded on the EMSA gel by the Smad3/4 complex and became more notable as the quantity of Smad3 increased (Fig. 1B). This finding indicated that Smad4 alone bound to the probe with low affinity, whereas the Smad3/4 complex enhanced the retardation more specifically. To eliminate nonspecific binding, unlabeled probes were used in a competitive EMSA assay. The retarded band was eliminated completely by a 100 or 200 -fold excess of unlabeled $\beta 3$ GalT4 promoter probe, whereas an unlabeled nonspecific probe, amplified by the forward and reverse pNon primers had no effect (Fig. 1C). Xia et al (17) reported that a DNA fragment (5'-GTCTAGAC-3') of the $\beta 3$ GalT4 promoter is a Smad4 binding site. To assess the relative contribution of the SBE, EMSAs were performed using an unlabeled probe containing either the intact SBE ( $\beta 3$ GalT4p) or a mutated sequence lacking SBE ( $\beta 3$ GalT4pm). The affinity of Smad3/4 for $\beta 3$ GalT4pm was eliminated completely compared with $\beta 3$ GalT4 $p$ (Fig. 1D). These findings indicated that the SBE is essential for Smad3/4 binding activity.

ChIP assays are commonly used to determine DNA-protein binding regions. In the present study, NMuMG cells were treated with TGF $\beta$ and formaldehyde was used to fix the cross-linking between the Smad3/4 proteins and their DNA target. The cross-linked DNA was extracted and fragmented by sonication and an anti-Smad4 antibody was used to screen the DNA fragments attached to the Smad4 protein. The forward and reverse $\beta 3 \mathrm{GalT} 4 \mathrm{pL}$ primers used in the above EMSAs were also used in the ChIP assays. PCR products of the correct size were obtained from the input DNA and the immunoprecipitated DNA, whereas no such PCR bands were detected in the control experiments, which used the same DNA fragment as the template, but without specific antibody addition. Negative control bands were amplified only with input DNA using the non-specific primers used in the EMSAs (Fig. 1E). Antibodies directed to Pol II, an enzyme responsible for transcription of protein-coding genes, were used as positive controls to ensure the accuracy of the ChIP results. Forward and reverse pactinp primers were used to detect the PCR band. Taken together, the findings from the EMSAs and ChIP assays indicated that the Smad $3 / 4$ complex bound specifically to the $\beta 3$ GalT4 promoter directly through the SBE.

Morphological alterations resulting from overexpression of Smad3 or Smad4. Our previous study demonstrated that the $\beta 3$ GalT4 gene, responsible for the expression of $\mathrm{Gg} 4$, is downregulated during the EMT process in NMuMG cells (9). Smads are key factors in this process. In the present study, to clarify the association between Smad3/4 and $\beta 3$ GalT4, Smad3 and Smad4 overexpression cells were constructed. NMuMG cells containing empty vector pcDNA3.1 were described as 'mock'.

The two transfectants, when cultured in normal DMEM, exhibited a flattened epithelial morphology similar to that of the mock (Fig. 2A), indicating that neither Smad3 nor Smad4 affected the expression of genes that control cell morphology. Exogenously added TGF $\beta$ activated the TGF $\beta /$ Smads signaling pathway and altered cell shape, with the transfectants and mock cells converted to a fibroblastic morphology (Fig. 2A).

Reduced expression of the epithelial marker E-cad and increased expression of the mesenchymal markers $\mathrm{N}$-cad and vimentin are characteristic processes of EMT. Cells overexpressing Smad3 and Smad4 demonstrated a markedly enhanced expression of vimentin and $\mathrm{N}$-cad and a reduced expression of E-cad and $\beta$-catenin, which forms a complex with E-cad stabilizing the cell-cell junction (Fig. 2B and C). TGF $\beta$ treatment intensified the enhanced and reduced expression of the EMT markers mentioned above. These findings suggested that the genes controlling these markers are regulated by Smads and involved in the TGF $\beta /$ Smads pathway via different regulatory mechanisms.

Level of $\beta 3$ GalT4 transcription in transfectants cells. To determine whether the $\beta 3$ GalT4 gene is positively or negatively regulated by Smad3/4, the transcription levels of $\beta 3$ GalT4 in two transfectants, as above, and mock were evaluated by semiquantitative and quantitative RT-PCR using the forward and reverse $\beta 3$ GalT4real primers. TGF $\beta$ treatment caused a reduction in $\beta 3$ GalT4 expression (Fig. 3A and B) and the degree of reduction was greater in the transfectants compared with the mock (Fig. 3B). These findings suggested that, following activation of the TGF $\beta /$ Smads signaling pathway by TGF $\beta$, the 
A

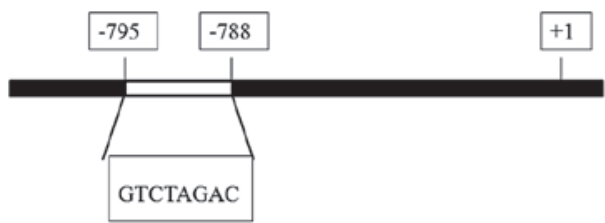

B

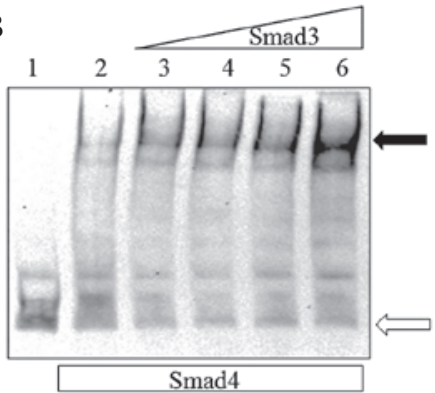

Unlabeled

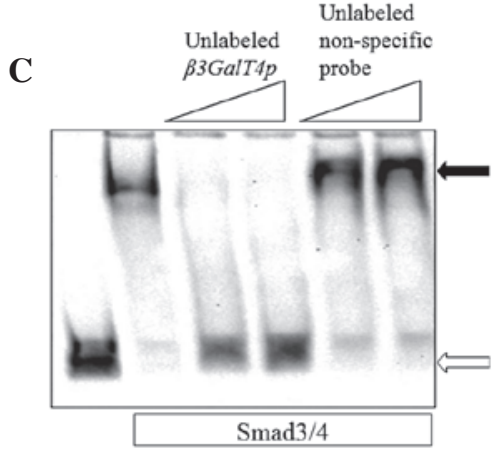

$\mathbf{E}$

D

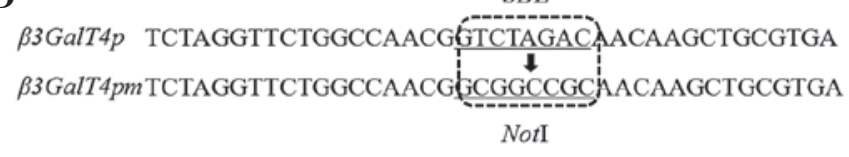
Unlabeled $\beta 3$ GalT $4 p$ Unlabeled $\beta 3$ GalT4pm

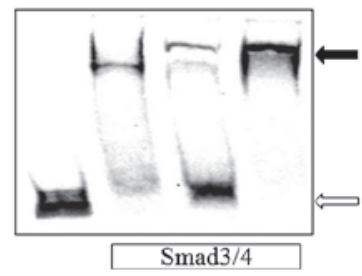

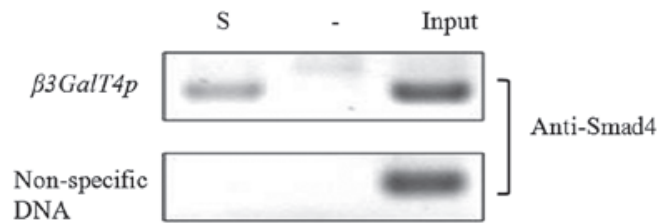

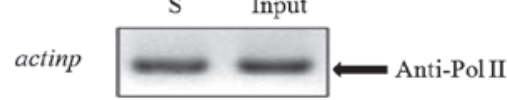

Figure 1. Analysis of Smad3/4 complex binding to the $\beta 3$ GalT4 promoter. (A) Smad4 binding site (5'-GTCTAGAC-3') located between positions -788 and -795, relative to the transcriptional start point of the $\beta 3$ GalT4 promoter. (B) EMSA. Each lane contained $0.3 \mathrm{nM}$ labeled probe. Smad4 protein (900 ng) was loaded. Smad3 (400, 600, 800 and $1000 \mathrm{ng}$ ) was loaded on lanes 3-6, respectively. (B-D) Black arrow indicates retarded DNA fragments; white arrow indicates free probes. (C) EMSA to eliminate the nonspecific binding of the Smad3/4 proteins. The labeled probe and 100 or 200 -fold excess of the unlabeled probe were used in competitive assays. A fragment of actin promoter was used as a nonspecific probe. (D) Determination of the binding sites of Smad3/4. Top: Nucleotide sequence of part of the $\beta 3$ GalT4 promoter region and SBE. All probes used were $40 \mathrm{bp}$. NotI sites were generated at the SBE site to produce a mutated probe. Underlined nucleotides were changed: Bottom: EMSA using the unlabeled probe and mutated or intact DNA fragment. (E) ChIP assays. Input DNA, a DNA fragment immunoprecipitated with anti-Smad4 antibody (lane ' $S$ ') and a DNA fragment immunoprecipitated with IgG (lanes '-') were used as templates for the polymerase chain reaction. A nonspecific DNA region was used as a negative control. Anti-Pol II antibodies that immunoprecipitated the Pol II-actin promoter complexes were used as a positive control. $\beta 3 \mathrm{GalT4}, \beta 1,3$-galactosyltransferase-4; EMSA, electrophoretic mobility shift assay; SBE, Smad4 binding element.
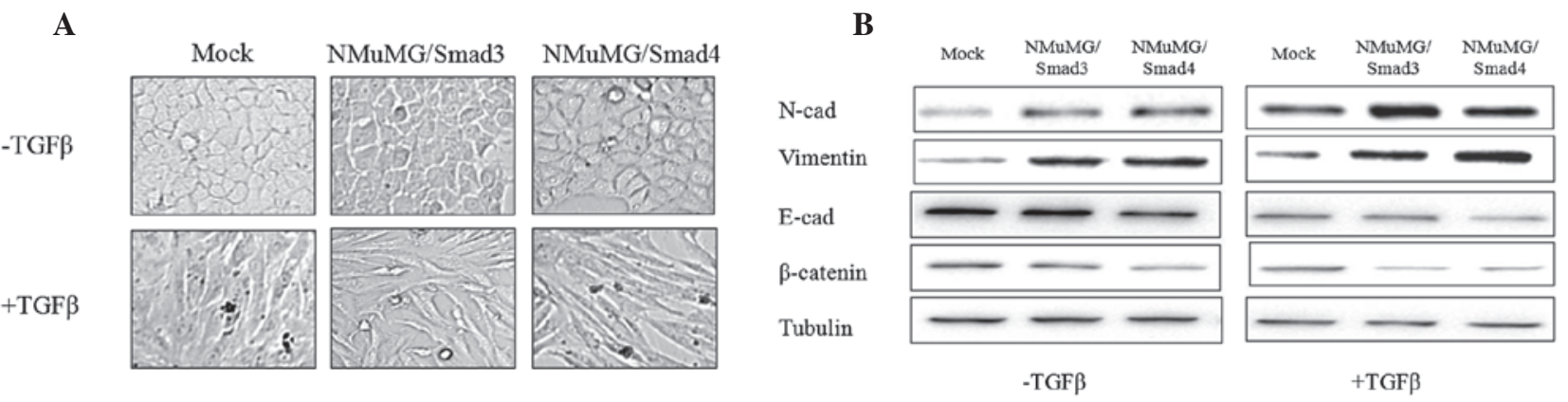

C
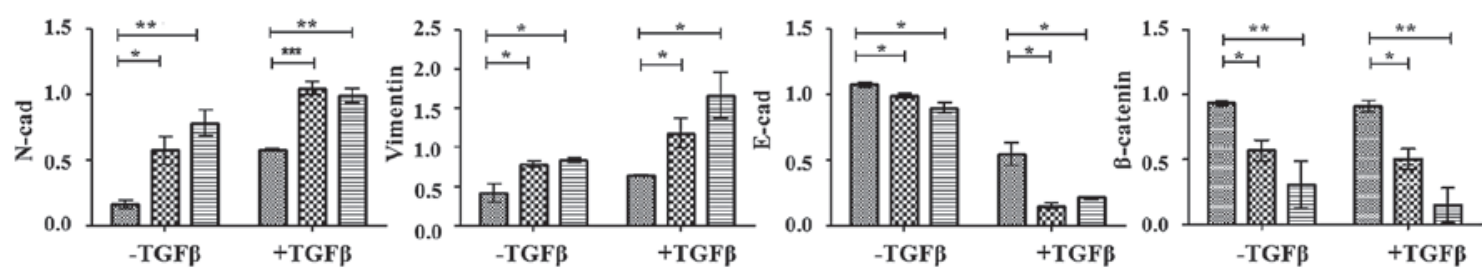

Mock

N NMuMG/Smad3

NMuMG Smad4

Figure 2. Effect of Smad3 or Smad4 overexpression in NMuMG cells. (A) Morphological changes. Cells were cultured in 6-well plates and treated with 2 ng/ $\mathrm{ml} \mathrm{TGF} \beta$ for $48 \mathrm{~h}$. Images were captured under phase-contrast microscopy. (B and C) Expression of EMT markers in the transfected cells. Cells were cultured in 6-well plates and treated with or without $2 \mathrm{ng} / \mathrm{ml}$ TGF $\beta$ for $48 \mathrm{~h}$. The cells were harvested and lysed in radioimmunoprecipitation assay buffer. Lysates (10 $\mu \mathrm{g}$ protein/well) were subjected to SDS-PAGE and western blot analysis and the expression of the markers N-cad, vimentin, E-cad and $\beta$-catenin was analyzed by western blot analysis, as described in Materials and methods. Representative western blot analysis results and expression levels relative to Tubulin are expressed as the mean \pm standard deviation from triplicate experiments. ${ }^{*} 0.01<\mathrm{P} \leq 0.05$ vs. mock. ${ }^{* * *} 0.001<\mathrm{P} \leq 0.01$ vs. mock; ${ }^{* * *} \mathrm{P} \leq 0.001$ vs. mock. NMuMG, normal murine mammary gland; TGF, transforming growth factor; E-cad, E-cadherin; N-cad, N-cadherin. 
$\mathbf{A}$

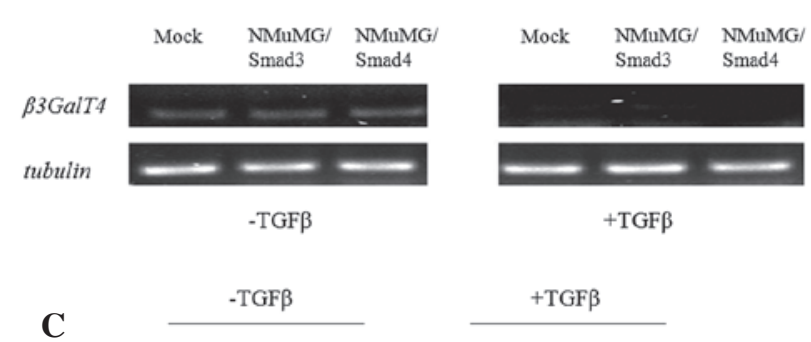

$\mathbf{C}$

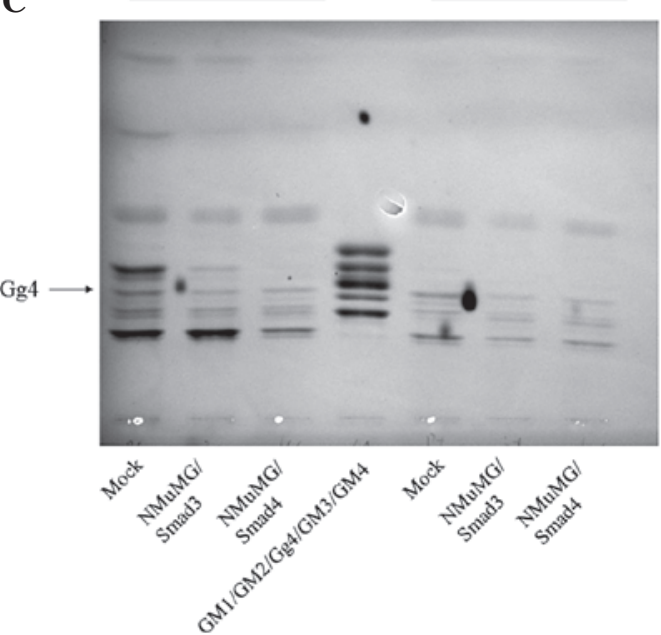

B

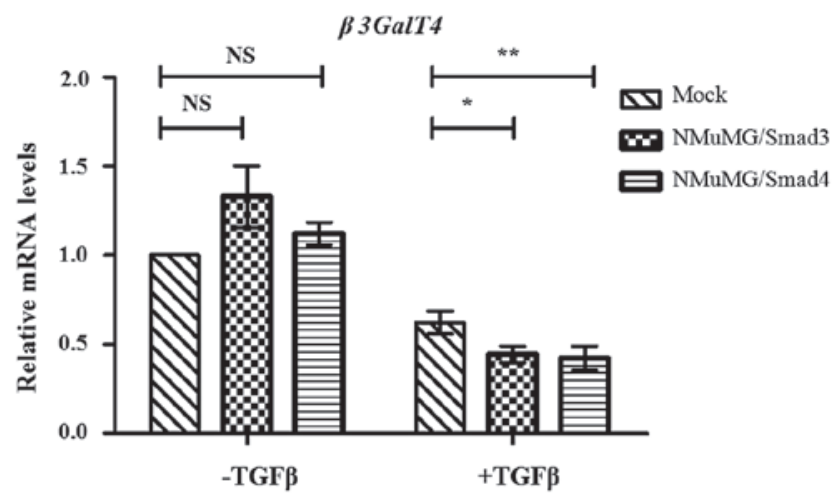

D

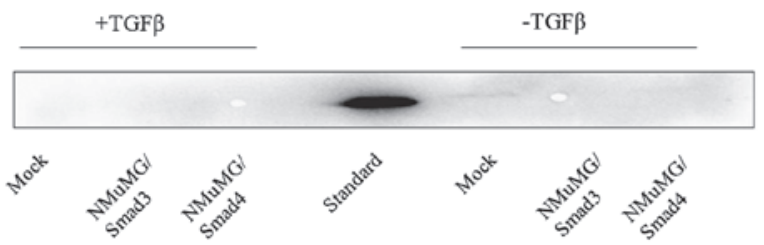

Figure 3. Expression of the $\beta 3$ GalT4 gene and Gg4 in Smads-overexpressing cells. Cells ( $2 \times 10^{5} /$ well) were cultured and treated with $2 \mathrm{ng} / \mathrm{ml} \mathrm{TGF} \beta$ for $48 \mathrm{~h}$. (A) Semiquantitative and (B) quantitative reverse transcription polymerase chain reactions were performed, as described in the Materials and methods. The housekeeping gene tubulin was used as a loading control. Data were analyzed using the $2^{-\Delta \Delta \mathrm{Ct}}$ method and expressed as fold change in gene expression relative to mock. NS, not significant ( $\mathrm{P}>0.05)$. ${ }^{*} 0.01<\mathrm{P} \leq 0.05$ vs. mock. ${ }^{* *} 0.001<\mathrm{P} \leq 0.01$ ( $\beta 3$ GalT4 gene vs. mock). (C and D) Cells $\left(\sim 2 \times 10^{6}\right)$ were cultured in $100 \mathrm{~mm}$ dishes and harvested. Total GSLs were extracted with isopropanol/hexane/water (55:25:20) for high performance thin layer chromatography detection and immunostained with TKH7. $\beta 3 \mathrm{GalT4}$, $\beta 1,3$-galactosyltransferase-4; Gg4, gangliotetraosylceramide; TGF, transforming growth factor; GM, Asialo-ganglio-N-tetraosylceramide; NMuMG, normal murine mammary gland.

Smads complex is translocated into the nucleus, binds to the 33 GalT4 promoter and downregulates its transcription.

Gg4 expression in transfectants cells. In our previous study, the mRNA levels of $\mathrm{Gg} 4$ and $\beta 3$ GalT4 were reduced during TGF $\beta$-induced EMT and the reduction in Gg4 was associated with decreased expression of E-cad and $\beta$-catenin (9). In the present study, total GSL fractions from transfectants and mock, with or without TGF $\beta$ treatment, were prepared and analyzed using HPTLC. The level of Gg4 expression decreased in parallel with the levels of E-cad and $\beta$-catenin (Fig. 3C and D). These findings suggested that the Smad3/4 complex affects the expression of Gg4 by suppressing mRNA levels of $\beta 3$ GalT4 and that Gg4 interacts closely with the E-cad/ $\beta$-catenin complex to stabilize the cell-cell junction.

\section{Discussion}

The enzyme $\beta 3 \mathrm{GalT} 4$ is responsible for the synthesis of $\mathrm{Gg} 4$ in the ganglioside biosynthetic pathway. The decreasing expression of its gene, $\beta 3$ GalT4, in the TGF $\beta$-induced EMT of NMuMG cells and in human breast cancer samples indicated its relevance to the formation and development of breast cancer (Table I). Therefore, the present study investigated the molecular mechanism underlying the reduced transcription of the $\beta 3$ GalT4 gene during TGF $\beta$-induced EMT. A $1.4 \mathrm{~kb}$ promoter sequence upstream of the $\beta 3$ GalT4 gene appears to include a number of potential binding sites for transcription factors, including Smad4 (17). In the present study, NMuMG cells, which undergo EMT when treated with TGF $\beta$, were used as a model to investigate the involvement of the Smad3/4 complex in the regulation of $\beta 3$ GalT4 expression. The results of EMSAs and ChIP assays demonstrated that the Smad3/4 complex bound directly to the $\beta 3$ GalT4 promoter (Fig. $1 \mathrm{~B}, \mathrm{C}$ and E). Sequence analysis by Xia et al revealed an SBE site (5'-GTCTAGAC-3') between positions -788 and -795 relative to the $\beta 3$ GalT4 transcriptional start point (17). In order to evaluate the role of this sequence in the interaction between Smads and DNA, an SBE mutated probe was generated for competitive EMSA (Fig. 1D). The results demonstrated that the SBE is important in Smads-DNA affinity binding.

Although Smads are key in the EMT process, NMuMG cells overexpressing either Smad3 or Smad4 did not exhibit notable morphological changes. These Smad3/4-overexpressing cells were highly sensitive to TGF $\beta$ treatment. Previous studies demonstrated that R-Smads and Smad4 constantly shuttle between the nucleus and cytoplasm, regardless of the presence or absence of a signal (20-22). An activated Smads complex is typically expressed at a low level to maintain normal physiological functions, and R-Smads are present in an unphosphorylated form without sensing signals (23). These findings may explain why the overexpression of Smads in NMuMG cells 
in the present study did not cause notable phenotypic changes (Fig. 2A). The overexpression of Smad3/4 did cause enhanced expression of the mesenchymal markers vimentin and $\mathrm{N}$-cad and reduced the expression of the epithelial marker E-cad and the intracellular signal transducer $\beta$-catenin (Fig. 2B and C). It appeared that EMT markers were regulated by the Smad transcription factors, as also indicated by previous studies (24-26). The direct or indirect mechanisms whereby Smad3/4 affect EMT markers remain to be elucidated.

Ourprevious study demonstrated that,during TGF $\beta$-induced EMT in NMuMG cells, the expression of the $\beta 3$ GalT4 gene and $\mathrm{Gg} 4$ are downregulated and $\mathrm{Gg} 4$ acts as an inhibitor of EMT, possibly by interacting with E-cad and $\beta$-catenin $(9,10)$. In the present study, the activated Smad3/4 complex was found to downregulate the expression of $\beta 3 \mathrm{GalT4}$ and the associated levels of mRNA in TGF $\beta$-treated cells (Fig. 3A and B). Consistent with this finding, the expression of $\mathrm{Gg} 4$, E-cad and $\beta$-catenin were all reduced in the TGF $\beta$-treated cells (Fig. 3C and D). Exogenous TGF $\beta$ presumably activated the Smads to form a complex and stimulated the translocation of this complex into the nucleus, where it bound to target genes, including $\beta 3$ GalT4, in the TGF $\beta /$ Smads signaling pathway.

In conclusion, the present study demonstrated that the activated Smad3/4 complex downregulated the expression of the $\beta 3$ GalT4 gene responsible for ganglioside expression through translocation into the nucleus and binding to the $\beta 3$ GalT4 promoter. The findings of the present study suggest that Gg4 is important in the TGF $\beta /$ Smads signaling pathway. Subsequent studies are required to assess this hypothesis and to elucidate the mechanisms whereby Gg4 and other GSLs are involved in the TGF $\beta$-mediated EMT processes.

\section{Acknowledgements}

This study was supported by the National Science Foundation for Young Scientists of China (grant no. 81201572), the Natural Science Foundation of Jiangsu Province, China (grant no. BK2012113), the Fundamental Research Funds for the Central Universities (grant no. JUSRP51319B) and Jiangsu Planned Projects for Postdoctoral Research Funds (grant no. 1201011C). The authors would like to thank Dr S. Anderson for editing the English of the manuscript.

\section{References}

1. Yamashita T, Wada R, Sasaki T, et al: A vital role for glycosphingolipid synthesis during development and differentiation. Proc Natl Acad Sci USA 96: 9142-9147, 1999.

2. Zeng G, Gao L, Birkle S and Yu RK: Suppression of ganglioside GD3 expression in a rat F-11 tumor cell line reduces tumor growth, angiogenesis, and vascular endothelial growth factor production. Cancer Res 60: 6670-6676, 2000.

3. Zeng G, Gao L and Yu RK: Reduced cell migration, tumor growth and experimental metastasis of rat F-11 cells whose expression of GD3-synthase is suppressed. Int J Cancer 88 : 53-57, 2000.
4. Hakomori S: Tumor malignancy defined by aberrant glycosylation and sphingo(glyco)lipid metabolism. Cancer Res 56: 5309-5318, 1996.

5. Hakomori S: Glycosylation defining cancer malignancy: new wine in an old bottle. Proc Natl Acad Sci USA 99: 10231-10233, 2002.

6. Allende ML and Proia RL: Lubricating cell signaling pathways with gangliosides. Curr Opin Struct Biol 12: 587-592, 2002.

7. Thiery JP, Acloque H, Huang RY and Nieto MA: Epithelial-mesenchymal transitions in development and disease. Cell 139: 871-890, 2009.

8. Quaggin SE and Kapus A: Scar wars: mapping the fate of epithelial-mesenchymal-myofibroblast transition. Kidney Int 80: 41-50, 2011

9. Guan F, Schaffer L, Handa K and Hakomori SI: Functional role of gangliotetraosylceramide in epithelial-to-mesenchymal transition process induced by hypoxia and by TGF-(beta). FASEB J 24: 4889-4903, 2010.

10. Guan F, Handa K and Hakomori SI: Specific glycosphingolipids mediate epithelial-to-mesenchymal transition of human and mouse epithelial cell lines. Proc Natl Acad Sci USA 106: 7461-7466, 2009.

11. Shi Y and Massagué J: Mechanisms of TGF-beta signaling from cell membrane to the nucleus. Cell 113: 685-700, 2003.

12. Xu L and Massagué J: Nucleocytoplasmic shuttling of signal transducers. Nat Rev Mol Cell Biol 5: 209-219, 2004.

13. Kang JS, Liu C and Derynck R: New regulatory mechanisms of TGF-beta receptor function. Trends Cell Biol 19: 385-394, 2009

14. Derynck $R$ and Zhang YE: Smad-dependent and Smad-independent pathways in TGF-beta family signalling. Nature 425: 577-584, 2003.

15. Zawel L, Dai JL, Buckhaults P, et al: Human Smad3 and Smad4 are sequence-specific transcription activators. Mol Cell 1: 611-617, 1998.

16. Shi Y, Wang YF, Jayaraman L, Yang H, Massagué J and Pavletich NP: Crystal structure of a Smad MH1 domain bound to DNA: insights on DNA binding in TGF-beta signaling. Cell 94: 585-594, 1998

17. Xia T, Gao L, Yu RK and Zeng G: Characterization of the promoter and the transcription factors for the mouse UDP-Gal:betaGlcNAc beta1,3-galactosyltransferase gene. Gene 309: 117-123, 2003.

18. Nelson EA, Walker SR, Alvarez JV and Frank DA: Isolation of unique STAT5 targets by chromatin immunoprecipitation-based gene identification. J Biol Chem 279: 54724-54730, 2004.

19. Todeschini AR, Dos Santos JN, Handa K and Hakomori SI: Ganglioside GM2-tetraspanin CD82 complex inhibits met and its cross-talk with integrins, providing a basis for control of cell motility through glycosynapse. J Biol Chem 282: 8123-8133, 2007.

20. Pierreux CE, Nicolás FJ and Hill CS: Transforming growth factor beta-independent shuttling of Smad4 between the cytoplasm and nucleus. Mol Cell Biol 20: 9041-9054, 2000.

21. Schmierer B and Hill CS: Kinetic analysis of Smad nucleocytoplasmic shuttling reveals a mechanism for transforming growth factor beta-dependent nuclear accumulation of Smads. Mol Cell Biol 25: 9845-9858, 2005.

22. Hill CS: Nucleocytoplasmic shuttling of Smad proteins. Cell Res 19: 36-46, 2009

23. Inman GJ, Nicolas FJ and Hill CS: Nucleocytoplasmic shuttling of Smads 2, 3, and 4 permits sensing of TGF-beta receptor activity. Mol Cell 10: 283-294, 2002.

24. Nieman MT, Prudoff RS, Johnson KR and Wheelock MJ: $\mathrm{N}$-cadherin promotes motility in human breast cancer cells regardless of their E-cadherin expression. J Cell Biol 147: 631-644, 1999.

25. Conacci-Sorrell M, Zhurinsky J and Ben-Ze'ev A: The cadherin-catenin adhesion system in signaling and cancer. J Clin Invest 109: 987-991, 2002.

26. Peinado H, Portillo F and Cano A: Transcriptional regulation of cadherins during development and carcinogenesis. Int J Dev Biol 48: 365-375, 2004. 